\title{
Compressible MHD in Spherical Geometry
}

\author{
Lorenzo Valdettaro and Maurice Meneguzzi \\ CERFACS, 42 Av. G. Coriolis, 31057 Toulouse CEDEX, France
}

\begin{abstract}
The generation of magnetic field by a conducting, compressible fluid inside a spherical shell is studied by direct numerical simulations. A pseudo-spectral method is used in order to resolve accurately all the scales present in the problem. The range of parameters considered is the following: a unit Prandtl number, Rayleigh numbers up to 100 times critical, Taylor number 625 , an aspect ratio of 2, a Mach number slightly less than 1, and pressure and temperature scale heights of the order of the thickness of the shell. A dynamo effect is observed for magnetic Prandtl numbers larger than 1 . We present the properties of the turbulent flow, the role of the helicity and of the differential rotation in the enhancement of the magnetic field, and the spectral properties of the flow fields.
\end{abstract}

\section{The reference state}

We consider a compressible fluid inside a spherical shell heated from within. We take as thermodynamic equation the perfect gas law. The equations are solved using a pseudo-spectral semi-implicit method (Valdettaro and Meneguzzi, 1989). No model is used for the small scales; the resolution used is 64 points in latitude, 128 points in longitude and 65 points in radius.

The reference state is determined as follows:

We suppose that the equilibrium quantities depend only on radius, and that the gravity is due to the inner core, i.e. we neglect the self-gravitation. Therefore the temperature, density and gravity profiles are of the form:

$$
g(r)=\frac{a_{0}}{r^{2}}, \quad T(r)=a_{1}+\frac{a_{2}}{r}, \quad \rho(r)=a_{3} T^{m}
$$

with $a_{0}, a_{1}, a_{2}$ and $a_{3}$ arbitrary constants; $m$ is the polytropic index. The constants are determined by giving the values of the following quantities:

The Rayleigh number

$$
R_{a} \equiv-\left.\frac{d S / C_{p}}{d r} \frac{g h^{3}}{\kappa \nu}\right|_{t o p}
$$

where $S$ is the entropy, $C_{p}$ is the specific heat at constant pressure, $h$ is the thickness of the shell, $g$ is the gravity and $\kappa$ and $\nu$ are the thermal conductivity 
and the kinematic viscosity. The Rayleigh number is the parameter which governs the onset of convection and of turbulent motions. We have taken $R_{a}=100 R_{a c}$, $R_{a c}$ being the critical Rayleigh number for the onset of convection.

The stratification rate $\chi \equiv \rho_{\text {top }} / \rho_{\text {bottom }}$. We take a constant density, i.e. $\chi=1$. Consequently the polytropic index $m$ is equal to zero.

The entropy gradient

$$
\varepsilon \equiv-\left.\frac{d S / C_{p}}{d \log r}\right|_{t o p} .
$$

This quantity measures the relative importance of compressibility, since it is proportional to the square of the Mach number (Gilman and Glatzmaier, 1981). We choose a value of order $1(\varepsilon=3)$ in order to have non negligible compressible effects. It turns out from the computations that the average Mach number is slightly less than 1.

The other quantities which define the initial state are:

The Taylor number $T_{a}=\Omega^{2} d^{4} / \nu^{2}$; we have taken a moderate rotation rate: $T_{a}=625$. The aspect ratio $\beta$, i.e. the ratio between the outer and the inner radius of the shell; we have chosen the value $\beta=2$. The Prandtl number: we have assumed the kinematic viscosity and the thermal diffusivity constant and equal. Therefore $P_{r} \equiv \nu / \kappa$ is equal to 1 . This assumption is in good agreement with the solar context if we assume that both $\nu$ and $\kappa$ there can be represented there as eddy diffusivities. Finally we have assumed a perfect monoatomic gas, i.e. $c_{p}=5 / 2$, $c_{v}=3 / 2$.

The boundary conditions we impose are the following: we specify the temperature at the bottom and at the top of the shell. The ratio between the inner and outer temperature is fixed by the parameters we have defined above: $T_{\text {bottom }} / T_{\text {top }}=1+2 \varepsilon \beta c_{p} /\left(c_{v}-m\right)=21$. We have thus between 2 and 3 temperature scale heights. For the velocity we consider rigid boundary conditions, i.e. $v=0$. Finally for the magnetic field we take a perfect conductor at the bottom of the shell (i.e. the radial components of the magnetic field and of the electric current vanish there) and we allow only for a radial component of the magnetic field at the top boundary.

\section{The properties of the flow}

We let the fluid evolve from the initial state defined above and without magnetic field until a statistically stationary state is achieved. We summarize here the characteristics of the flow we obtain.

The entropy profile, averaged in latitude and longitude, is shown in Fig. 1. As expected, the flow field acts to maintain a constant entropy inside the shell. The central adiabatic part is surrounded by the inner and outer thermal boundary layers, which represent roughly $10 \%$ of the total width. In Fig. 2 we plot the density profile. We find, as in Massaguer and Zahn (1980), a reversal of the density perturbation near the top of the shell, indicating a negative buoyancy force there. This effect does not exist in the incompressible case. 
The efficiency of the convection is measured by the Nusselt number which is the ratio of the total superadiabatic energy flux divided by the purely conductive energy flux. The profile we obtain is strongly dependent on the radius, going from a minimum of about 3 near the boundaries to a maximum value of 15 at a middle radius.

The effects of compressibility are measured by the average Mach number, which in our case is around 0.3. The maximum Mach number fluctuates in time around a value of 0.6 . The flow is therefore entirely subsonic. The Reynolds number averaged over the sphere is around 60 , with a maximum value of 140 . The Rossby number $R_{o}=R_{e} / \sqrt{T_{a}}$, which measures the importance of rotation is slightly less than 1 .

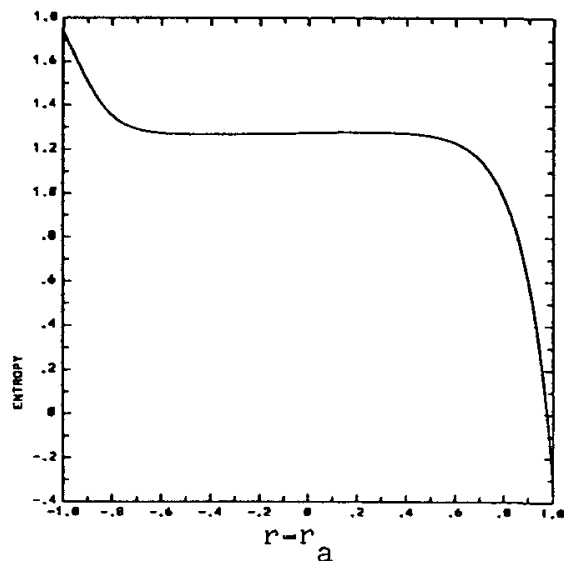

Figure 1. Radial profile of the entropy, averaged over the angles. Note the central adiabatic part.

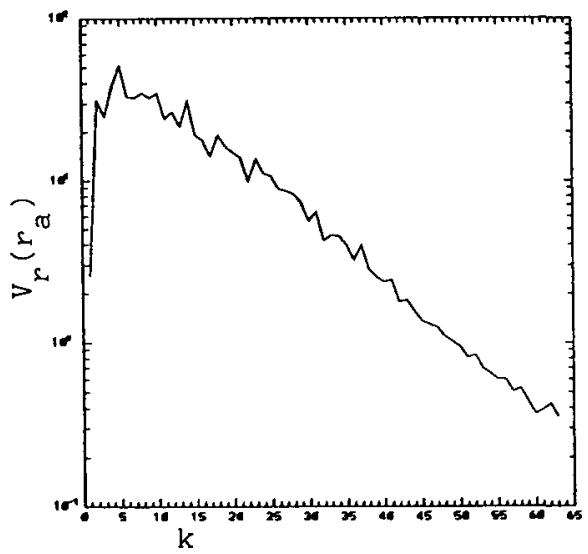

Figure 3. Horizontal spectrum of the radial component of the velocity at the middle radius.

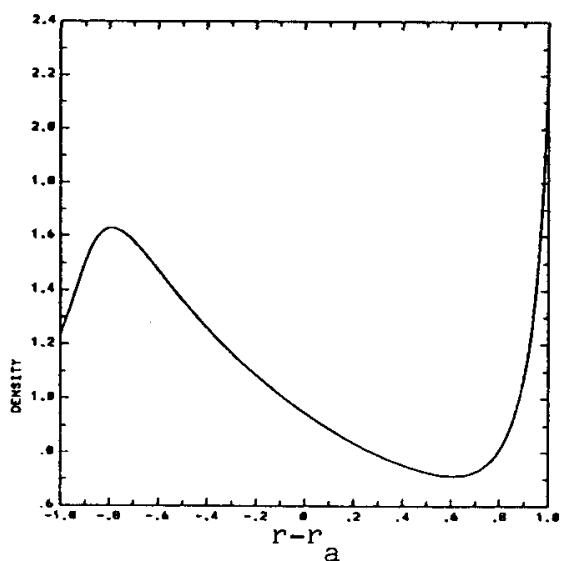

Figure 2. Radial profile of the density, averaged over the angles. Note the reversal of the density perturbation near the top of the shell.

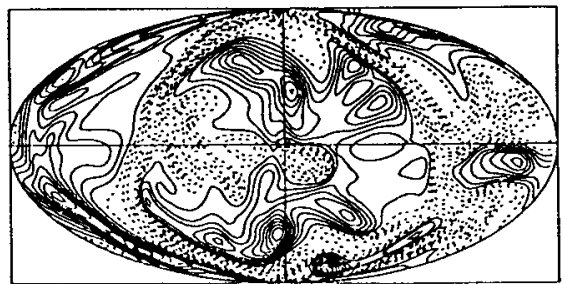

Figure 4. Mollweide projection on the sphere of the isocontours of the radial velocity at middle radius. Continuous lines: $V_{r}$ pointing toward the exterior; broken lines: $V_{r}$ pointing toward the interior. 


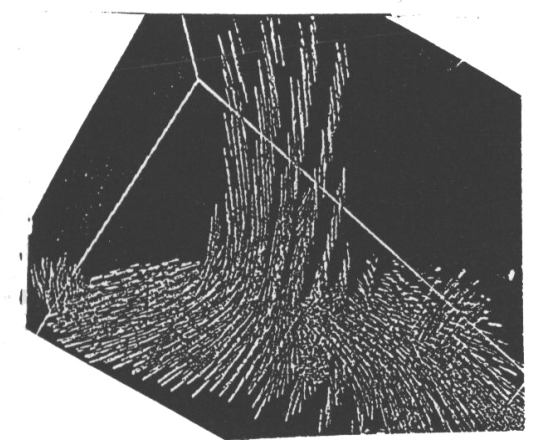

Figure 5. View of a descending plume. The velocity vectors are represented by a vector of length proportional to the velocity. Only vectors larger than a given threshold are shown. The outer part of the shell is on top.

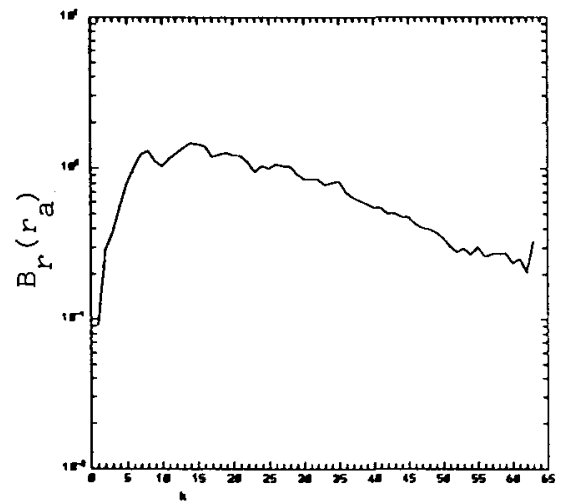

Figure 7. Horizontal spectrum of the radial magnetic field at middle radius.

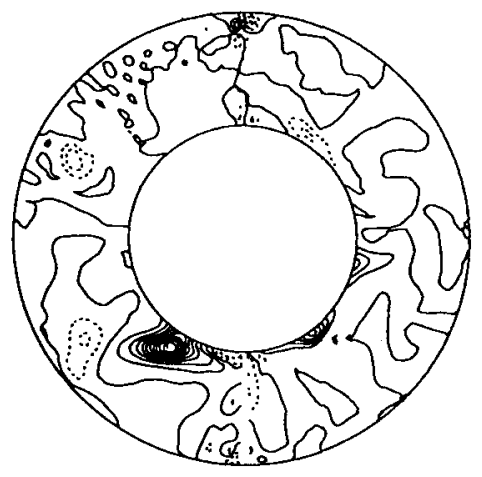

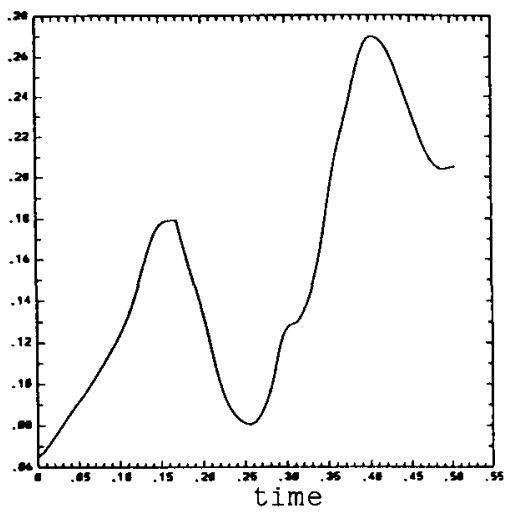

Figure 6. Evolution in time of the total magnetic energy during the nonlinear phase.

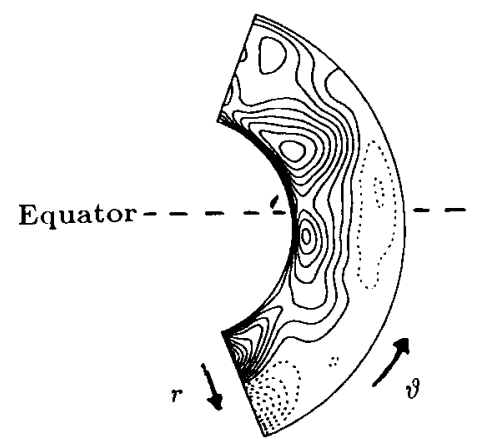

Figure 8. Isocontours of the differential rotation. Continuous lines: rotation in the direction of the mean rotation. Broken lines: rotation in the opposite direction of the mean rotation.

Figure 9. Isocontours of the toroidal component of the magnetic field. Cut in a meridional plane. Note the strong magnetic tube at the base of the convection zone. 
The fluid displays motions in a wide range of scales; this is clearly seen from the spectrum of the radial velocity, which is plotted in Fig. 3. The large scale motions are organised in the form of convection cells. In Fig. 4 are shown the isocontours of radial velocity at the middle radius. We see that there are both ascending and descending cells; however, the most intense cells are descending. In Fig. 5 is shown a descending plume: it has the form of a thin tube, enlarged at the base, and extending throughout the depth of the shell.

\section{The dynamo effect}

After the statistically stationary state has developed we have introduced a seed magnetic field of very low intensity $\left(E_{m} / E_{k i n} \simeq 10^{-5}\right)$ and we let it evolve in time. At the beginning we have frozen the fluid and we let the magnetic field evolve alone. The equation for the magnetic field becomes linear and the evolution is exponential in time (it is the "kinematic dynamo"). We find, for a magnetic Prandtl number $P_{m}=2.5$, an intensification of the magnetic field with a small growth rate, of the order of the thermal diffusion time. We have thus a slightly supercritical dynamo effect. Higher values of the magnetic Prandtl number would have lead to stronger dynamo effects, but we could not increase $P_{m}$ above that value, without increasing the resolution of the computations.

We have then let the fluid evolve together with the magnetic field for 0.4 thermal times, which is much larger than the eddy turnover time (of the order of $0.03 t_{t h}$ in our calculations) but it is only of the order of the growth rate of the magnetic field we expect from the linear analysis. In Fig. 6 we plot the evolution of the magnetic energy in time. We clearly see an increase, which is however much lower than those observed in other direct simulations (see e.g. Meneguzzi and Pouquet, 1989; Brandenburg et al., 1991). Although we have not reached the stage of the nonlinear saturation of the magnetic field, we are able to explore and interpret the growth phase.

In Fig. 7 is plotted the spectrum of the radial component of the magnetic field, at middle radius. As expected (Meneguzzi et al., 1981) the magnetic field spectrum appears to be more flat than that of the velocity field (Fig. 3). This means that the magnetic field displays structures on smaller scale than the velocity field.

The two major factors which tend to increase the magnetic field are the differential rotation $\left\langle v_{\phi}\right\rangle / r \sin \vartheta$, which generates strong toroidal magnetic field, and helicity $\langle\boldsymbol{v} \cdot \boldsymbol{\omega}\rangle$, which generates poloidal fields from toroidal fields. We plot in Fig. 8 a typical profile of the differential rotation we obtain. We clearly see a strong generation of differential rotation near the base of the convection zone. As expected, this strong differential rotation generates a strong toroidal magnetic field. This is shown in Fig. 9, where the contour lines of $B_{\phi}$ are plotted in a meridional plane. We see the section of a strong magnetic tube at the base of the convection zone.

The relative helicity $\left(H \equiv\langle\boldsymbol{v} \cdot \omega\rangle /\left(\left\langle v^{2}\right\rangle\left\langle\omega^{2}\right\rangle\right)^{1 / 2}\right)$ in our run fluctuates between a minimum of $1 \%$ and a maximum of $6 \%$. This value is small, although of the same order of magnitude of that found in Meneguzzi and Pouquet (1989) in 
Boussinesq approximation, and could explain the small growth rate we obtain for the magnetic field.

In conclusion, our three-dimensional simulation suggests a consistent compressible dynamo, based at the bottom of the convection zone, probably due to an $\alpha-\Omega$ mechanism. This mechanism (Parker, 1955) has been the most successful one for the solar dynamo. Applications of our results to the solar context are however not possible, due to the large difference between the solar parameters and those we can afford with present computers. We think that the interest of such direct simulations is to give a better insight to the basic mechanisms and lead to a better theoretical understanding of the dynamo problem.

\section{References}

Brandenburg, A., Jennings, R., Nordlund, Å., Stein, R.F., Tuominen, I.: 1991, these Proceedings

Gilman, P.A., Glatzmaier, G.A.: 1981, Astrophys. J. Suppl. 45, 335

Massaguer, J.M., Zahn, J.P.: 1980, Astron. Astrophys. 87, 315

Meneguzzi, M., Frisch, U., Pouquet, A.: 1981, Phys. Rev. Letters 47, 1060

Meneguzzi, M., Pouquet, A.: 1989, J. Fluid Mech. 205, 297

Parker, E.N.: 1955, Astrophys. J. 121, 491

Valdettaro, L., Meneguzzi, M.: 1989, in Proceedings from the Workshop on Supercomputing Tools for Science and Engineering, Pisa 